Chirurg 2017 $\cdot 88: 71$

DOI 10.1007/s00104-016-0331-x

Online publiziert: 7. Dezember 2016

o) Springer Medizin Verlag Berlin 2016

CrossMark

\section{H. Dralle · F. Weber}

Sektion Endokrine Chirurgie, Klinik für Allgemein-, Viszeral- und Transplantationschirurgie,

Universitätsklinikum Essen, Essen, Deutschland

\title{
Aktive Überwachung vs. primäre Chirurgie beim papillären Mikrokarzinom der Schilddrüse
}

\section{Originalpublikation}

Oda H, Miyauchi A, Ito $\mathrm{Y}$ et al (2016)

Incidences of unfavorable events in the management of low-risk papillary microcarcinoma of the thyroid by active surveillance versus immediate surgery. Thyroid 26: 150-155

Hintergrund und Fragestellung. In den letzten zwei Jahrzehnten kam es weltweit $\mathrm{zu}$ einer deutlichen Zunahme der kleinen papillären Schilddrüsenkarzinome [1]. Neben der Frage nach der Ursache dieses Phänomens, steht vor allem deren therapeutische Konsequenz im Mittelpunkt zahlreicher Diskussionen. Verschiedene Studien, vor allem des japanischen Kuma-Hospitals [2, 3] stellen dar, dass papilläre Mikrokarzinome (PTMC) nicht in jedem Fall primär operiert werden müssen, sondern den Patienten auch eine primäre Observationsstrategie angeboten werden kann [4]. In der vorliegenden Untersuchung werden $2153 \mathrm{~Pa}$ tienten mit Low-risk-PTMC (LRPTMC) vorgestellt, die zwischen 2005 und 2013 im Kuma-Hospital, Japan, entweder primär aktiv beobachtet (1179) oder primär operiert (974) wurden.

Material und Methoden. Die Diagnose eines PTMC wurde durch Feinnadelpunktion gestellt. Eine primär observierende Strategie wurde nur denjenigen Patienten angeboten, die Primärtumoren ohne High-risk-Kriterien (Metastasen, extrathyreoidales Tumorwachstum, Dedifferenzierung oder Primärtumor nahe Trachea bzw. N. recurrens) hatten. Patienten, die eine primär aktive
Überwachung wählten, wurden nach 6 Monaten, dann jährlich nachuntersucht. Die Nachbeobachtungszeit lag bei einem Median von 47 Monaten.

Ergebnisse. Die primäre Chirurgie erfolgte als Hemithyreoidektomie mit paratrachealer Lymphknotendissektion (575) oder totale Thyreoidektomie mit zentraler Lymphknotendissektion (399). Von 1179 primär aktiv beobachteten Patienten wurden 94 (8,0\%) sekundär operiert. Die Gründe hierfür waren geänderter Patientenwunsch (54\%), eine Größenzunahme des Tumors (29\%) oder das Auftreten von Lymphknotenmetastasen (6\%).

Zwischen den beiden Behandlungsgruppen (primär aktive Überwachung vs. primäre Chirurgie) wurden folgende signifikanten Unterschiede festgestellt: passagere Stimmlippenparese $0,6 \%$ vs. 4,1 \%; passagere Hypokalzämie 2,8 \% vs. $16,7 \%$; permanenter Hypoparathyreoidismus $0,08 \%$ vs. 1,6\%; Thyroxinsubstitution $20,7 \%$ vs. $66,1 \%$; postoperatives Hämatom $0 \%$ vs. $0,5 \%$; chirurgische Narbe $8,0 \%$ vs. $100 \%$. Keine signifikanten Unterschiede fanden sich bei den Parametern permanente Stimmlippenparese, zervikales Rezidiv und Tod.

Diskussion und Fazit. Die vorliegenden retrospektiven Studien werden als hinreichende Grundlage dafür gewertet, dass unter Einhaltung bestimmter Kriterien Patienten mit LRPTMC ein primär observierendes Vorgehen angeboten werden kann, da die onkologischen Resultate sich nicht unterscheiden und die operativ bedingte Morbidität bei primärer $\mathrm{Ob}$ - servation deutlich geringer ist. Die japanischen Observationsstudien haben die weltweite Diskussion über die therapeutische Strategie bei intrathyreoidalen papillären Schilddrüsenkarzinomen in hohem Maße beeinflusst und erneut das diskursive Pendel in die Richtung eines „weniger ist mehr“ verschoben. Ob dies auch für Zentraleuropa Berechtigung hat, wo die meisten PTMC als pathohistologische Zufallsbefunde diagnostiziert werden, müssen jetzt entsprechende Studien zeigen.

\section{Korrespondenzadresse}

Prof. Dr. med. Dr. h. c. mult., FRCS, FACS, FEBS H. Dralle

Sektion Endokrine Chirurgie, Klinik für Allgemein-, Viszeral- und Transplantationschirurgie, Universitätsklinikum Essen

Hufelandstr. 55, 45147 Essen, Deutschland

henning.dralle@uk-essen.de

Interessenkonflikt. H. Dralle und F. Weber geben an, dass kein Interessenkonflikt besteht.

\section{Literatur}

1. Ahn HS et al (2014) Korea's thyroid-cancer „epidemic" - screening and overdiagnosis. NEngl J Med 371:1765-1767

2. Ito $Y$ et al (2003) An observation trail without surgical treatment in patients with papillary microcarcinoma of the thyroid. Thyroid 13:381-387

3. Ito $Y$ et al (2014) Patient age is significantly related to the progression of papillary microcarcinoma of the thyroid under observation. Thyroid 24:27-34

4. Brito JPetal (2016) A clinical framework to facilitate risk stratification when considering an active surveillance alternative to immediate biopsy and Surgery in papillary microcarcinoma. Thyroid 26:144-149 